\title{
Gastritis enfisematosa en asociación con un adenocarcinoma gástrico, a propósito de un caso
}

\author{
Emphysematous gastritis in association with gastric adenocarcinoma. \\ A case report
}

Esteban Vanegas-Duarte,,$^{1 *}$ (1] Angélica María Duque-Montaño. ${ }^{2}$

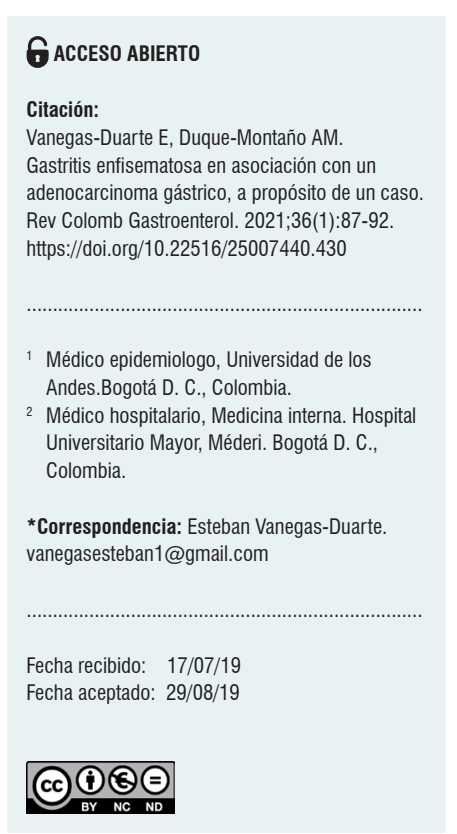

\begin{abstract}
Resumen
A través del presente reporte se describe un caso de gastritis enfisematosa, una rara condición clínica consistente en la invasión de la pared gástrica por microorganismos productores de gas. Puede resultar en una situación fatal debido a lo inespecífico de su clínica y a lo tórpido de su evolución. En este caso se describe a un paciente anciano de 77 años, con alta fragilidad, quien cursaba con un cuadro de gastritis enfisematosa que no respondió a manejo médico y requirió gastrectomía de urgencia. Adicionalmente, cursaba con un adenocarcinoma gástrico ulcerado infiltrante, que previamente no había sido diagnosticado, como probable factor condicionante y desencadenante.
\end{abstract}

\section{Palabras clave}

Gastritis enfisematosa, enfisema gástrico, neoplasias gástricas, infecciones por Clostridium.

\section{Abstract}

This report describes a case of emphysematous gastritis, a rare clinical condition consisting of invasion of the gastric wall caused by gas-producing bacteria. It can lead to fatal outcomes due to the unspecific nature of the symptoms and its torpid course. The following is the case of a highly fragile 77-year-old male patient, who presented with symptoms of emphysematous gastritis that did not respond to medical treatment and required emergency gastrectomy. In addition, the patient had an infiltrating ulcerated gastric adenocarcinoma, which had not previously been diagnosed and was a probable conditioning and triggering factor.

\section{Keywords}

Emphysematous gastritis, Gastric emphysema, Gastric neoplasms, Clostridial infections.

\section{INTRODUCCIÓN}

El presente documento contiene el primer caso de gastritis enfisematosa reportado y publicado en Colombia. La gastritis enfisematosa es una desconocida entidad con una alta tasa de letalidad causada por la producción de gas en la pared estomacal debido a la invasión de gérmenes en las capas profundas de la cámara gástrica (1). No existen consensos de manejo integral y su abordaje se basa en experiencias documentadas a través de los escasos reportes de caso. Posiblemente, el extendido desconocimiento condiciona una importante tasa de subdiagnóstico, lo que deriva en intervenciones tardías y un aumento exponencial de la mortalidad. El diagnóstico precoz permite intervenciones tempranas que facilitan el abordaje y reducen el impacto clínico $(1,2)$. Los últimos reportes parecen avalar una conducta conservadora, no quirúrgica en primera instancia, con resultados favorables (2). Nuestro objetivo cardinal es narrar la experiencia clínica en un caso nunca visto en nuestra institución y desconocido para la mayoría de nosotros; 
no obstante, se obtuvo un diagnóstico radiológico precoz que permitió orientar la terapia.

\section{CASO CLÍNICO}

Se trataba de un paciente de 76 años, masculino, con antecedentes de enfermedad de Alzheimer avanzada e hipertensión arterial. Ingresó por un cuadro clínico de 12 horas de evolución consistente en dolor abdominal intenso que inició en la región epigástrica, con irradiación a mesogastrio, tipo cólico y asociado con picos febriles no cuantificados. Negaba emesis, cambios en las deposiciones u otra sintomatología asociada. A la revisión por sistemas, su hija manifestaba pérdida no voluntaria de peso ( 7 kilos en 3 meses, aproximadamente). Al ingreso se apreciaba un paciente taquicárdico y febril, con tendencia a la hipotensión, con marcada distensión abdominal y dolor a la palpación generalizada, palidez generalizada, frialdad distal y signos leves de dificultad respiratoria. Se indicó la toma de analítica de ingreso y se encontró una marcada leucocitosis y neutrofilia, función renal alterada y gases arteriales que evidenciaban acidosis metabólica grave con trastorno moderado de la oxigenación e hiperlactatemia (Tabla 1). Se establece un puntaje SOFA de ingreso de 6 puntos. Se indicó la toma de una tomografía de urgencia que evidenciaba la sobredistensión de cámara gástrica con neumatosis intestinal, portograma aéreo y engrosamiento de la pared gástrica con burbujas de gas en su interior (Figura 1).

Ante la sospecha de gastritis enfisematosa se inició una antibioticoterapia de amplio espectro con piperacilina/ tazobactam y metronidazol. Se ingresó el paciente a la unidad de cuidado intensivo (UCI) y se iniciaron maniobras de reanimación, fluidoterapia y el cubrimiento antibiótico previamente descrito. A las 48 horas de ingreso, aproximadamente, la evolución del paciente inició tórpida y se evidenciaron signos de irritación peritoneal e inestabilidad hemodinámica. A los pocos minutos de evidenciar los signos de irritación peritoneal, fue llevado a laparotomía de urgencia con hallazgos de necrosis de la pared gástrica y peritonitis purulenta de los 4 cuadrantes. Se realizó gastrectomía total con anastomosis en Y de Roux y lavado de la cavidad abdominal. El paciente egresó de sala de cirugía a la UCI con soporte vasopresor y ventilación mecánica invasiva. Sus parámetros hemodinámicos obligaron a instaurar

Tabla 1. Paraclínicos relevantes en la analítica de ingreso del paciente

Pruebas de laboratorio de ingreso

\begin{tabular}{|c|c|c|c|}
\hline \multicolumn{4}{|c|}{ Pruebas de laboratorio de ingreso } \\
\hline Paraclínico & Valor del paciente & Método de análisis & Rangos de normalidad \\
\hline Nitrógeno ureico & $68 \mathrm{mg} / \mathrm{dL}$ & UV cinético & $<20 \mathrm{mg} / \mathrm{dL}$ \\
\hline Creatinina sérica & $1,8 \mathrm{mg} / \mathrm{dL}$ & Colorimétrico enzimático & $0,5 \mathrm{mg} / \mathrm{dL}-1,0 \mathrm{mg} / \mathrm{dL}$ \\
\hline Recuento de leucocitos & $18,4 \times 10^{3} / \mu \mathrm{L}$ & Microscopia automatizada & $5,10-9,70 \times 10^{3} / \mu \mathrm{L}$ \\
\hline Neutrófilos absolutos & $17,6 \times 10^{3} / \mu \mathrm{L}$ & Microscopia automatizada & $1,40-6,50 \times 10^{3} / \mu \mathrm{L}$ \\
\hline Linfocitos absolutos & $0,9 \times 10^{3} / \mu \mathrm{L}$ & Microscopia automatizada & $1,20-3,40 \times 10^{3} / \mu \mathrm{L}$ \\
\hline Hto & $25,3 \%$ & Microscopia automatizada & $38,0 \%-47,0 \%$ \\
\hline $\mathrm{Hb}$ & $9,1 \mathrm{~g} / \mathrm{dL}$ & Microscopia automatizada & $12-15 \mathrm{~g} / \mathrm{dL}$ \\
\hline Plaquetas & $151 \times 10^{3} / \mu \mathrm{L}$ & Láser de semiconducción & $150-450 \times 10^{3} / \mu \mathrm{L}$ \\
\hline Transaminasa alcalina & $54 \mathrm{UI} / \mathrm{L}$ & Cinético & $5-60 \mathrm{UI} / \mathrm{L}$ \\
\hline AST & $26 \mathrm{UI} / \mathrm{L}$ & Cinético & 10 a $34 \mathrm{UI} / \mathrm{I}$ \\
\hline Fosfatasa alcalina & $102 \mathrm{UI/L}$ & Cinético & 44 a 147 UI/L \\
\hline ELISA para VIH & $0,15 \mathrm{UI} / \mathrm{mL}$ & ELISA de cuarta generación & $0,0-0,9 \mathrm{UI} / \mathrm{mL}$ \\
\hline Bilirrubina & $1,5 \mathrm{mg} / \mathrm{dL}$ & Fotocolorimetría & $0,2-1,2 \mathrm{mg} / \mathrm{dL}$ \\
\hline Lactato & $3,6 \mathrm{mmol} / \mathrm{L}$ & Espectrofotométrico & $0,1-1,5 \mathrm{mmol} / \mathrm{L}$ \\
\hline
\end{tabular}

AST: aspartato-aminotransferasa; ELISA: enzimoinmunoanálisis de adsorción; Hto: hematocrito; Hb: hemoglobina; VIH: virus de la inmunodeficiencia humana. 

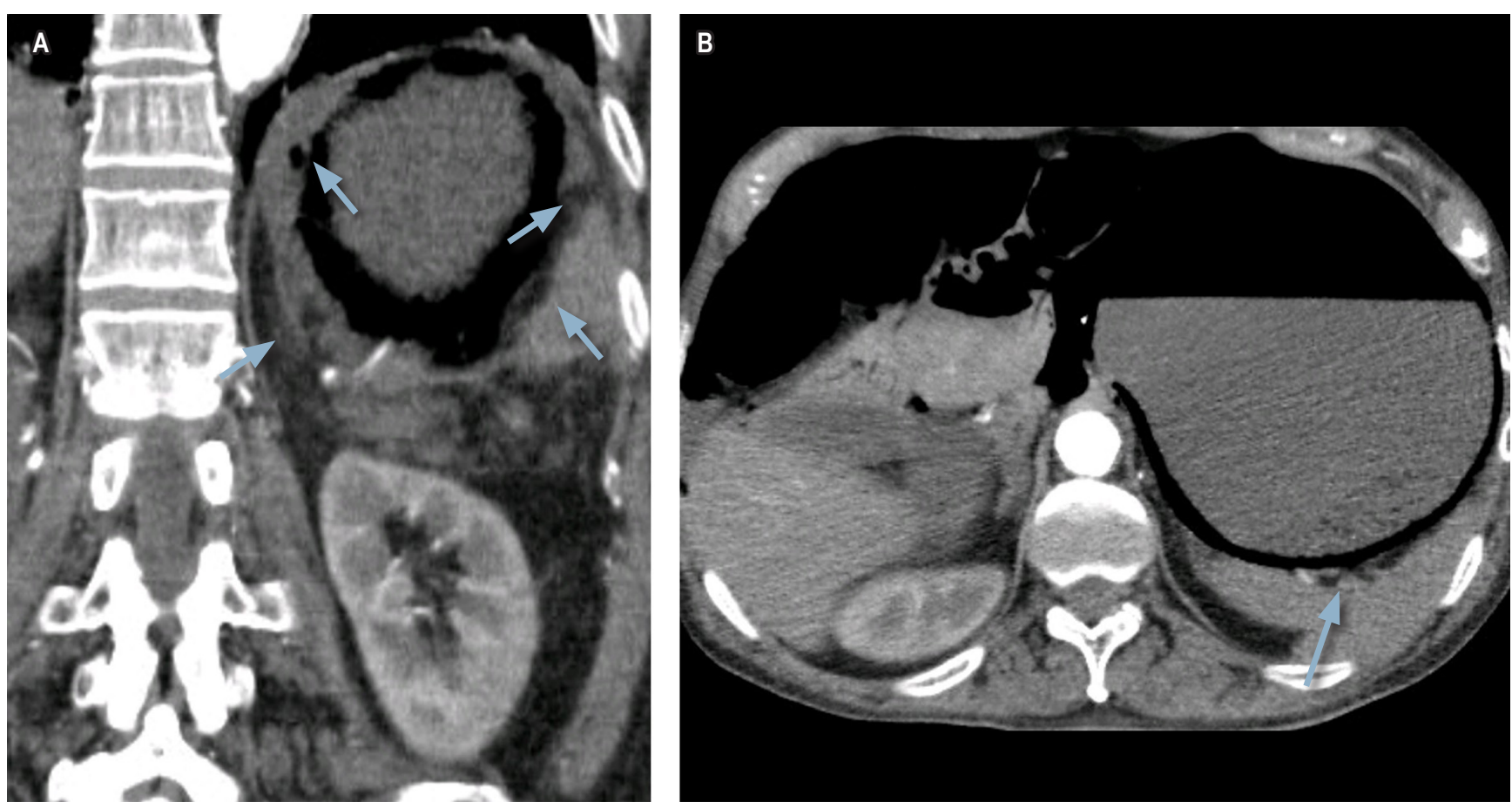

Figura 1. Tomografía abdominal con contraste del paciente. Se observa sobredistensión de la cámara gástrica y hallazgos compatibles con infiltración de gas en las paredes del estómago. Las flechas señalan la presencia de burbujas de gas en la pared estomacal.

una infusión de noradrenalina y vasopresina. A las pocas horas, por hipotensión refractaria, el paciente requirió el inicio de dobutamina y al escalonamiento del manejo antibiótico a carbapenémico y glucopéptido.

Una analítica de control mostró la persistencia de leucocitosis y neutrofilia, el empeoramiento de la función renal y la nula depuración del lactato (Tabla 2). Después del inicio de antibioticoterapia de amplio espectro, el paciente tuvo un período de estabilidad de aproximadamente 72 horas, pero 4 días después de la laparotomía, el paciente presentó un paro cardiorrespiratorio. Se documentó ritmo de asistolia. Luego de 20 minutos de maniobras de reanimación, se determinó el fallecimiento. El reporte histopatológico documentó numerosas colonias bacterianas submucosas de tipo Clostridium sp., gastritis enfisematosa y lesión compatible con adenocarcinoma gástrico infiltrante.

\section{DISCUSIÓN}

La gastritis enfisematosa es una rara infección generada por la invasión de bacterias productoras de gas a la pared gástrica que da lugar a inflamación supurativa con compromiso progresivo de las capas profundas, formación de abscesos y subsecuente necrosis (3). Es una entidad con alta mortalidad debido a lo inespecífico de su clínica. Este factor ocasiona retrasos en el diagnóstico y en la oportuna terapia (3). Pocos son los casos publicados en la literatura y existe un amplio desconocimiento de la naturaleza de la enfermedad. La fisiopatología de la gastritis enfisematosa no se encuentra establecida $(1,4)$. Se ha considerado que las lesiones en la mucosa permiten la penetración de microorganismos a planos profundos de la pared (4). También se ha sugerido que puede ser secundario a diseminación por vía hematógena desde un foco a distancia (4). Los síntomas son inespecíficos e incluyen un amplio espectro de manifestaciones como el dolor abdominal de predominio en el epigástrico, náuseas, emesis y diarrea (4, $5)$. Algunos autores establecen un signo patognomónico: emesis de un coágulo mucoso necrótico que sigue la forma de la pared gástrica secundario a la disección de la mucosa muscular (5). Generalmente, al momento del diagnóstico los pacientes cursan con signos de respuesta inflamatoria como fiebre y leucocitosis o, inclusive, se presentan con inestabilidad hemodinámica (5).

En este punto es necesario realizar una distinción entre enfisema gástrico y gastritis enfisematosa: dos entidades que se confunden con facilidad y que en gran parte de las ocasiones se consideran una misma patología, aunque su comportamiento clínico resulta diametralmente opuesto. A través de revisiones sistémicas de la literatura se ha logrado 
Tabla 2. Paraclínicos relevantes en la analítica tomada en el posoperatorio durante la estancia del paciente en la UCI

\begin{tabular}{llll}
\hline \multicolumn{1}{c}{ Paraclínico } & \multicolumn{1}{c}{ Valor del paciente } & \multicolumn{1}{c}{ Método de análisis } & Rangos de normalidad \\
\hline Nitrógeno ureico & $76 \mathrm{mg} / \mathrm{dL}$ & UV cinético & $<20 \mathrm{mg} / \mathrm{dL}$ \\
\hline Creatinina sérica & $2,7 \mathrm{mg} / \mathrm{dL}$ & Colorimétrico enzimático & $0,5 \mathrm{mg} / \mathrm{dL}-1,0 \mathrm{mg} / \mathrm{dL}$ \\
\hline Recuento de leucocitos & $21,3 \times 10^{3} / \mu \mathrm{L}$ & Microscopia automatizada & $5,10-9,70 \times 10^{3} / \mu \mathrm{L}$ \\
Neutrófilos absolutos & $20,2 \times 10^{3} / \mu \mathrm{L}$ & Microscopia automatizada & $1,40-6,50 \times 10^{3} / \mu \mathrm{L}$ \\
\hline Linfocitos absolutos & $0,6 \times 10^{3} / \mu \mathrm{L}$ & Microscopia automatizada & $1,20-3,40 \times 10^{3} / \mu \mathrm{L}$ \\
\hline Hto & $18,2 \%$ & Microscopia automatizada & $38,0-47,0 \%$ \\
\hline Hb & $7,1 \mathrm{~g} / \mathrm{dL}$ & Microscopia automatizada & $12-15 \mathrm{~g} / \mathrm{dL}$ \\
\hline Plaquetas & $80 \times 10^{3} / \mu \mathrm{L}$ & Laser de semiconducción & $150-450 \times 10^{3} / \mu \mathrm{L}$ \\
\hline Bilirrubina & $2,1 \mathrm{mg} / \mathrm{dL}$ & Fotocolorimetría & $0,2-1,2 \mathrm{mg} / \mathrm{dL}$ \\
\hline Lactato & $5,5 \mathrm{mmol} / \mathrm{L}$ & Espectrofotométrico & $0,1-1,5 \mathrm{mmol} / \mathrm{L}$
\end{tabular}

dilucidar y caracterizar cada patología de forma más o menos precisa. Matsushima y colaboradores realizaron una interesante revisión en la que lograron una distinción clara que permitió la formulación de criterios que facilitan la diferenciación entre ambas condiciones al momento de abordar un paciente con clínica y hallazgo radiológico de gas en la pared gástrica (6). Esta diferenciación se logró a través de la observación de 73 casos reportados (6). El enfisema gástrico es un escenario en el cual se identifica acumulación de gas en la pared estomacal. Ocurre como consecuencia de la entrada de aire al interior de la pared secundario a disrupciones en la mucosa gástrica (6). Se estableció que el enfisema gástrico tiene mayor relación con lesiones mecánicas directas de la mucosa o con desgarros por aumento de presiones intraluminales (7). El $41 \%$ de los casos de enfisema gástrico cursa con neoplasias gástricas subyacentes (6). Una vez se corrige o se trata el daño de la mucosa, el pronóstico del enfisema gástrico suele ser favorable $(6,7)$. El enfisema gástrico puede dar lugar a dolor abdominal de diferentes intensidades que se suele acompañar de náuseas y emesis. En la mayoría de los casos, los síntomas se resuelven sin requerir intervenciones clínicas e incluso existen pacientes asintomáticos cuyo diagnóstico es incidental y producto del estudio de otras condiciones como tumores gástricos.

En contraste, los aproximadamente 40 casos reportados en la literatura anglosajona de gastritis enfisematosa demuestran que esta entidad es producto de la invasión de microorganismos productores de gas a la pared gástrica y que no se relaciona necesariamente con alteraciones en la integridad de la mucosa $(6,8)$. La revisión de la literatura realizada por Watson y colaboradores en 2016 estableció que en casi la mitad de los casos reportados no se han identificado los microorganismos causantes; en el $20 \%$ se aislaron varios gérmenes, considerándose una etiología polimicrobiana; y en el $6 \%$ se encontró la presencia de bacterias de la especie Clostridium (8). En el resto de los casos se hallaron múltiples especies de bacterias, parásitos como el estrongiloides y hongos como la Candida y el Mucor (6, $7)$. Se han identificado posibles factores predisponentes tales como el consumo de alcohol, la diabetes mellitus, la desnutrición y el linfoma $(8,9)$.

Algunos autores han descrito la diferenciación imagenológica entre enfisema gástrico y gastritis enfisematosa. Se ha descrito que el hallazgo tomográfico más importante en el enfisema gástrico consiste en bandas lineales de aire que siguen la disposición de las capas de la pared gástrica. En el enfisema gástrico no suele existir engrosamiento de la mucosa (10). No existe un estándar de oro para el diagnóstico de la gastritis enfisematosa. Se ha propuesto que la sospecha clínica en combinación con la radiografía simple de abdomen es suficiente para orientar un diagnóstico (8). Sin embargo, el amplio desconocimiento de la enfermedad podría dar lugar a que esta combinación no sea suficiente para soportar un diagnóstico cercano. La tomografía de abdomen posee mayor sensibilidad y especificidad para la detección de gas intramural (2). Los hallazgos imagenológicos que se aprecian en las tomografías de pacientes con 
gastritis enfisematosa incluyen quistes de aire que infiltran la pared de la cámara gástrica, neumoperitoneo y gas en el sistema portal $(4,8)$. También se describe engrosamiento de la pared de la cámara gástrica con edema de la submucosa y abscesos intramurales $(8,11)$. Es más común el hallazgo de neumatosis intestinal y neumoperitoneo en la gastritis enfisematosa que en el enfisema gástrico (10).

En aproximadamente el $45 \%$ de los casos reportados de pacientes con evidencia de aire en la pared gástrica se ha realizado endoscopia de vías digestivas altas (6). Más de la mitad de los casos de gastritis enfisematosa que han sido llevados a endoscopia demuestran necrosis de la pared del estómago $(6,10)$. Adicionalmente, se han descrito zonas de eritema y edema con mucosa friable o áreas ulceradas con exudado fibrinopurulento (12). En el enfisema gástrico no se han descrito hallazgos de necrosis o exudado purulento; en esta entidad es común el hallazgo de eritema y edema sin otras alteraciones. En la gastritis enfisematosa los estudios histopatológicos revelan engrosamiento mucoso con infiltración de leucocitos y trombosis vascular (13). El estudio patológico en ocasiones permite la observación del microorganismo causante de la entidad (12).

En cuanto al tratamiento de la gastritis enfisematosa, las revisiones han demostrado que la cirugía inmediata no parece ser la elección conveniente como primera línea de manejo y su realización debe estar considerada en aquellos pacientes con perforación e isquemia $(14,15)$. Algunos reportes de caso han mostrado la efectividad de la terapia antibiótica de amplio espectro asociada con terapias de soporte como la reanimación hídrica $(7,8)$. La cirugía no ha demostrado conferir beneficios adicionales y no garantiza mejores resultados de supervivencia (8). Al ser causada por múltiples tipos de microorganismos, la gastritis enfisematosa debe ser tratada con antibioticoterapia de amplio espectro, en lo posible conjugada, y debe considerar la prevalencia de los principales microrganismos descritos en los distintos reportes de casos $(8,15,16)$. La cirugía será reservada para aquellos casos en los que el paciente curse con signos de irritación peritoneal, inestabilidad hemodinámica, choque séptico u otros hallazgos sugerentes de perforación gástrica y peritonitis subsecuente (13).

En los casos en que la diferenciación entre enfisema gástrico y gastritis enfisematosa no se logre, Matsushima y colaboradores propusieron un algoritmo de abordaje para el paciente con clínica y hallazgos radiológicos de aire en la pared gástrica (6). Al abordar un paciente con dolor abdominal y hallazgos de gas intramural Matsushima y colaboradores propusieron el inicio de fluidoterapia, manejo antibiótico de amplio espectro, descompresión con sonda nasogástrica y restricción de la vía oral (6). Si aún persisten dudas de diagnóstico, se sugiere la realización de endoscopia de vías digestivas altas (6). Si existe visualización de isquemia o necrosis de la pared estomacal, lo más conveniente es la realización de laparotomía (6).

\section{REFERENCIAS}

1. Singh K. Emphysematous Gastritis Associated with Sarcina ventriculi. Case Rep Gastroenterol. 2019;13(1):207-213. https://doi.org/10.1159/000499446

2. Jehangir A, Rettew A, Shaikh B, Bennett K, Qureshi A, Jehangir Q. A case report of emphysematous gastritis in a diabetic patient: favorable outcome with conservative measures. J Community Hosp Intern Med Perspect. 2015;5(4):28010. https://doi.org/10.3402/jchimp. v5.28010

3. Al-Jundi W, Shebl A. Emphysematous gastritis: case report and literature review. Int J Surg. 2008;6(6):e63-6. https:// doi.org/10.1016/j.ijsu.2007.02.007

4. Weaver A, Weintraub R, Smith B. Recognizing emphysematous gastritis. JAAPA. 2019;32(2):27-29. https://doi. org/10.1097/01.JAA.0000550284.27345.5e

5. Goyal O, Bansal A, Goyal P. Emphysematous Gastritis with Air in Portal Venous System. J Assoc Physicians India. 2017;65(5):105-106.

6. Matsushima K, Won EJ, Tangel MR, Enomoto LM, Avella DM, Soybel DI. Emphysematous gastritis and gastric emphysema: similar radiographic findings, distinct clinical entities. World J Surg. 2015;39(4):1008-17. https://doi. org/10.1007/s00268-014-2882-7

7. Curelli J, Fridenberg G, Cereceda S, García SJE. Enfisema gástrico benigno versus gastritis enfisematosa. Revista del Hospital Privado de Comunidad. 2004;7(1):43-46.

8. Watson A, Bul V, Staudacher J, Carroll R, Yazici C. The predictors of mortality and secular changes in management strategies in emphysematous gastritis. Clin Res Hepatol Gastroenterol. 2017;41(1):e1-e7. https://doi. org/10.1016/j.clinre.2016.02.011

9. Alvarado-Lezama J, Espinosa-González O, García-Cano E, Sánchez-Córdova G. Gastritis enfisematosa secundaria a mucormicosis gástrica. Cir Cir. 2015;83(1):56-60. Spanish. https://doi.org/10.1016/j.circir.2015.04.025

10. Johnson PT, Horton KM, Edil BH, Fishman EK, Scott WW. Gastric pneumatosis: the role of CT in diagnosis and patient management. Emerg Radiol. 2011;18(1):65-73. https://doi.org/10.1007/s10140-010-0888-5

11. Jung C, Choi YW, Jeon SC, Chung WS. Acute diffuse phlegmonous esophagogastritis: radiologic diagnosis. 
AJR Am J Roentgenol. 2003;180(3):862-3. https://doi. org/10.2214/ajr.180.3.1800862

12. Lee BS, Kim SM, Seong JK, Kim SH, Jeong HY, Lee HY, Song KS, Kang DY, Noh SM, Shin KS, Cho JS. Phlegmonous gastritis after endoscopic mucosal resection. Endoscopy. 2005;37(5):490-3. https://doi. org/10.1055/s-2005-861254

13. Rada Palomino A, Muñoz-Duyos A, Pérez Romero N, Vargas Pierola H, Puértolas Rico N, Ruiz Campos L, Espinós Pérez J, Veloso Veloso E. Gastritis flemonosa: una entidad poco frecuente como diagnóstico diferencial del abdomen agudo. Descripción de un caso y revisión bibliográfica. Rev Esp Enferm Dig. 2014;106(6):418-424.

14. Takano Y, Yamamura E, Gomi K, Tohata M, Endo T, Suzuki R, Hayashi M, Nakanishi T, Hanamura S, Asonuma K, Ino
S, Kuroki Y, Maruoka N, Nagahama M, Inoue K, Takahashi $\mathrm{H}$. Successful conservative treatment of emphysematous gastritis. Intern Med. 2015;54(2):195-8. https://doi. org/10.2169/internalmedicine.54.3337

15. Zamora Elson M, Labarta Monzón L, Escos Orta J, Cambra Fierro P, Vernal Monteverde V, Seron Arbeloa C. Gastritis enfisematosa, eficacia del tratamiento con antibioterapia precoz. Gastroenterol Hepatol. 2016;39(6):393-5. https:// doi.org/10.1016/j.gastrohep.2015.05.008

16. Nemakayala DR, Rai MP, Rayamajhi S, Jafri SM. Role Of Conservative Management In Emphysematous Gastritis. BMJ Case Rep. 2018;2018:bcr2017222118. https://doi. org/10.1136/bcr-2017-222118 